\title{
Chronic exposure to low doses of ozone produces a state of oxidative stress and blood-brain barrier damage in the hippocampus of rat
}

\author{
Selva Rivas-Arancibia ${ }^{1}$, Luis Fernando Hernández-Zimbrón ${ }^{1}$, Erika Rodríguez-Martínez ${ }^{1}$, \\ Gabino Borgonio-Pérez ${ }^{1}$, Varsha Velumani ${ }^{1}$, Josefina Durán-Bedolla ${ }^{2}$ \\ ${ }^{1}$ Departamento de Fisiología, Facultad de Medicina, Universidad Nacional Autónoma de México, México D. F., México \\ ${ }^{2}$ Centro de Investigación Sobre Enfermedades Infecciosas, Instituto Nacional de Salud Pública, Cuernavaca, México \\ Email: ${ }^{*}$ srivas@unam.mx
}

Received 26 September 2013; revised 25 October 2013; accepted 3 November 2013

Copyright (C) 2013 Selva Rivas-Arancibia et al. This is an open access article distributed under the Creative Commons Attribution License, which permits unrestricted use, distribution, and reproduction in any medium, provided the original work is properly cited.

\begin{abstract}
Chronic exposure to low doses of ozone similar to a day of high pollution in Mexico City causes a state of oxidative stress. This produces a progressive neurodegeneration in hippocampus of rats exposed to the gas. The aim of this study was to analyze the effect of chronic exposure on the changes in the blood-brain barrier in rats exposed to low doses of ozone. Method: each group received one of the following treatments, control group received air without ozone, and groups $2,3,4,5$, and 6 received ozone doses of $0.25 \mathrm{ppm}$ for 4 h daily during $7,15,30,60$ and 90 days respectively. Each group was processed to inmunohistochemical technique against of the following antibody: bloodbrain barrier, guanylyl cyclase, Iba-1, GFAP, NF $\kappa-B$, TNF- $\alpha$. The results show that there is a correlation between the time exposure of ozone and the progressive damage, on the blood-brain barrier rupture, finally causing edema of endothelial cell, increase in guanylyl cyclase type 1 , thickening of the processes and astrocytes foot, and an increase in the expression of factors NF $\kappa$-B and TNF- $\alpha$ at 30 and 60 days of exposure to this gas. All the above indicates that the chronic state of oxidative stress causes a neurodegeneration process, accompanied by disruption of the blood-brain barrier likely to occur in the Alzheimer's disease.
\end{abstract}

Keywords: Ozone; Oxidative Stress; Blood-Brain Barrier; Neurodegenerative Diseases

\section{INTRODUCTION}

The environmental contamination is a major health prob-

"Corresponding author. lem in the densely populated and industrialized cities $[1,2]$. The ozone, which is a product of the photochemical air pollution, is the principal contaminant [3]. High levels of air contamination have been associated with an increase and deteriorating of chronic degenerative respiratory illness and also cardiopulmonary and neurodegenerative disease [4-6].

It is well established that an acute as well as chronic inhalation of this gas produces a state of oxidative stress. When a redox imbalance occurs by an acute exposition to high doses of ozone ( $0.8-1.0 \mathrm{ppm})$ for $4 \mathrm{~h}$, it causes cell damage, accompanied with swelling, inflammation and changes in mitochondrial as in the endoplasmic reticulum occurs [6,7]. All these changes are reversible, since the increase in reactive oxygen species (ROS) in the organisms induces the stimulation of antioxidants systems which increases their activities [7]. In addition these systems also have the reparation function that permits to revert the pathological changes that have been found. The changes in the redox balance are also produced during the respiratory burst by the activation of immune system as a response of an acute defense of the organism against the pathogens [8]. Once the infection has been handed over the balance of the oxide reduction will be recovered. However, the chronic oxidative stress state is present in degenerative diseases; this state is characterized by a chronic increase of reactive oxygen species (ROS), reactive nitrogen species (RNS), and reactive species of transition metals [9]. In this state, the antioxidant systems are unable to revert the oxidative damage and as they are progressing it also alters the intracellular signaling. As well as other physiological regulatory systems such as the loss of regulation of the inflammatory response, this contributes to increase even more the oxidative stress state. Also there is a progressive mitochon- 
drial damage and alteration which produces a deficit of ATP [7]. Oxidative stress causes cell damage which will not permit the cells to maintain homeostasis together with the alteration in the intracellular signaling. It also activates phosphorylation pathway and inhibits the dephosphorylating pathway. Altogether, these changes finally lead to neuronal death. Some characteristics of the chronic neurodegenerative disease are the loss of the regulation of inflammatory response, progression of pathophysiological complications which takes it to a loss in the cognitive functions, progressive neuronal death and an incapability of cerebral reparation mechanisms (since the process of neurogenesis is blocked by the oxidative stress) [6] which causes the neurodegeneration process to progress.

Prolonged exposure to ozone creates a chronic oxidative stress state; in our laboratory we have developed a progressive neurodegeneration animal model. When the animals are exposed chronically to low doses of ozone similar to those of a high contaminated day in Mexico City $(0,25 \mathrm{ppm}$ for $4 \mathrm{~h}$ a day during $7,15,30,60$ and 90 days), with an increase of ozone exposure days, we observed a rise in oxidized lipids and proteins, the expression of $\mathrm{Cu} / \mathrm{Zn}$ superoxide dismutase increases, however its activity reduces. It also has been found that the glutathione system is altered (article in preparation); with this model, we show that a chronic disease caused by an oxidative stress state after 30 days of ozone exposure becomes to be irreversible. All these changes go along with the loss of the regulation of inflammatory response. During the progressive neurodegeneration, besides the neuronal changes, it also produces astrocytes alteration and activated microglia [6]. In this model, we demonstrated that in healthy animals with no other factor added; only oxidative stress, caused by low doses of ozone is capable to trigger a process of progressive neurodegeneration in the hippocampus of rats. Furthermore, this process becomes irreversible after 30 days of exposure and neurodegeneration continues to progress in relation to time.

In addition, the brain antioxidant systems are at a disadvantage in comparison with other organs such as the liver, pancreas and lungs. Brain has very low levels of catalase, moderated levels of superoxide dismutase despite its high content of phospholipids and high consumption of oxygen. However, the brain has a strict regulation of the compounds that may be in contact with neurons through blood-brain barrier (BBB) [10]. The selectivity of the $\mathrm{BBB}$ is vital to brain tissue, since it prevents toxic substances from getting easily in contact with the nerve cells [11]. This is because the BBB is formed by endothelial cells, pericytes and astrocytes that make it impermeable to water and permeable to very small lipid molecules, due to the above, the cells that form the barrier have specific proteins which allow the passage of small molecules like oxygen, glucose and micronutrients through it $[11,12]$.

BBB properties change according to the needs of the brain and exist in a close relationship between neurons, microglia, astrocytes and BBB for which it is considered that they form a neurovascular unit which also includes immune system cells [10]. The barrier has the ability to respond to changes, both inside and outside the CNS, it is also capable of secreting or inducing the secretion of substances, in some cases such as in Alzheimer's disease it has been found an elevated activity of nitric oxide synthase [13]. Our aim is to study the effect of chronic oxidative stress on the $\mathrm{BBB}$ in the hippocampus of rats exposed to low doses of ozone.

\section{MATERIALS AND METHODS}

\subsection{Materials}

Monoclonal mouse antiglial fibrillar acidic protein (GFAP) and polyclonal mouse anti-ionized calcium-binding adapter molecule 1 (Iba-1) antibodies were obtained from Biocare, polyclonal mouse anti-guanylyl cyclase and anti-BBB antibodies were obtained from Abcam. Mouse polyclonal anti-nuclear factor kappa-B (NF $\kappa$-B) was obtained from Santa Cruz Biotechnology, CA, USA.

\subsection{Animals}

Animal care and handling were done according to the Norma Official Mexicana NOM-036-SSA2-2002, the National Institutes of Health guidelines for Animal Treatment and the Ethics Committee of the Facultad of Medicina at the Universidad Nacional Autónoma de México. Thirty six male Wistar rats weighing 250 - $300 \mathrm{~g}$ were individually housed in acrylic boxes with free access to water and food (Purina, Minnetonka, MN) and kept in a clear air room.

They were randomly divided into six experimental groups: group 1) exposed to an air stream free of ozone during 30 days; group 2) exposed for 7 days to ozone; group 3) exposed for 15 days to ozone; group 4) exposed for 30 days to ozone; group 5) exposed for 60 days to ozone and group 6) exposed for 90 days to ozone. Ozone exposure was done daily for $4 \mathrm{~h}$ with a dose of $0.25 \mathrm{ppm}$. Immediately after the exposure to ozone, animals were returned to their home cages.

\subsection{Ozone Exposure}

Animals were put daily, for $4 \mathrm{~h}$, inside a chamber with a diffuser connected to a variable-flux ozone generator ( 5 $1 / \mathrm{s})$. The procedure used has been described elsewhere (Rivas-Arancibia et al., 2010). The air feeding the ozone converter was filtered purified air. Ozone production levels were proportional to the current intensity and to the airflow. A PCI Ozone \& Control System Monitor 
(PCI Ozone \& Control Systems, West Caldwell, NJ) was used to measure the ozone concentration inside the chamber during the experiment and to keep the ozone concentration constant.

Air exposure: The same chamber was used for treating the control group where a flow of ozone-free purified air was used.

\subsection{Immunohistochemistry Techniques}

After the completions of the treatments, animals from each group were anesthetized with sodium pentobarbital (50 $\mathrm{mg} / \mathrm{kg}$ ip) and intracardially perfused with $4 \%$ paraformaldehyde (Sigma-Aldrich, St Louis, MO). In 0.1M phosphate buffer (PB) pH 7.4 (Tecsiquim, México), the brains were postfixed with $10 \%$ formaldehyde for $24 \mathrm{~h}$ and embedded in paraffin (Merck, Darmstadt, Germany). Sagittal brain slices containing the hippocampus of these animals were cut at $5 \mu \mathrm{m}$ on a microtome, mounted on slides, and processed for immunohistochemistry. The immunohistochemistry for GFAP, NF $\kappa$-B, Iba-1, Guanylyl cyclase, tumor necrosis factor $\alpha(\mathrm{TNF}-\alpha)$ and $\mathrm{BBB}$ was done as follows: Slices of each brain containing the hippocampus had the paraffin removed, treated with heat-retrieval solution (Biocare Medical, Concord, CA), and put into an electric pressure cooker (Decloaking Chamber; Biocare Medical) for 5 min. After being washed with distilled water and treated with hydrogen peroxide (J.T. Baker) (diluted 1:5) for $5 \mathrm{~min}$, the slices were rinsed again and treated with a blocking reagent (Background Sniper; Biocare Medical) for $10 \mathrm{~min}$. They were washed with PBS (Merck), pH 7.4, and incubated overnight at 4 $\mathrm{C}$ with anti-GFAP (purified monoclonal mouse antibody, diluted 1:200; Biocare), anti-NF $\kappa$-B (purified polyclonal mouse antibody, diluted 1:200), anti-Iba-1 (purified polyclonal goat antibody, diluted 1:300), anti-guanylyl cyclase (purified polyclonal mouse antibody, diluted 1:200), anti-TNF- $\alpha$ or anti-BBB (purified monoclonal mouse antibody, diluted 1:200).

Sections were rinsed with PBS and treated with a secondary antibody using Trekkie Universal Link (Starr Trek Universal HRP Detection; Biocare Medical) for $1 \mathrm{~h}$. The sections were later washed with PBS and then treated with Trekavidin-HRP Label (Starr Trek Universal HRP Detection; Biocare Medical) for $30 \mathrm{~min}$ or the bound antibody was visualized using 3, 3-diaminobenzidine (DAB Substrate Kit; ScyTek, Logan, UT) as the chromogen. The slices were washed with distilled water and contrasted with hematoxylin buffer solution (ScyTek, Concord, CA.). For double immunofluorescence, following primary antibody incubation the sections were washed with TBS and incubated with the appropriate Alexa, 488, or 594-conjugated secondary antibody (Invitrogen).
Representative brain slices from each group were processed in parallel. After cover slipping with Entellan (F/550 ml; Merck), the slices were examined with an Olympus BX41 Microscope (Olympus, Japan) using a $100 \times$ magnification and photographed (Evolution VF-FCLR-12, Media Cybernetics camera; Bethesda, MD).

\section{RESULTS}

\subsection{Double Fluorescent Immunohistochemistry against Blood-Brain Barrier and Guanylyl Cyclase}

The results show that the blood-brain barrier damage increases with the time of exposure to ozone as shown in Figure 1. We can also observe alterations in the endothelial cells that are expressing guanylyl cyclase. There is an increase in the expression of this enzyme after sixty days of exposure as well as an increase in endothelial cells volume and at 90 days of exposure to ozone, BBB and endothelial cells are completely destroyed.

\subsection{Double Fluorescent Immunohistochemistry against Astrocytes and Microglia}

We found an increase in the immunoreactivity of Iba-1 and GFAP protein. The results indicate the activation of both microglia and astrocytes with respect to exposure time.

\subsection{Immunohistochemistry against astrocytes, NF $\kappa$-B and TNF- $\alpha$}

The results show an increase in the thickness of the

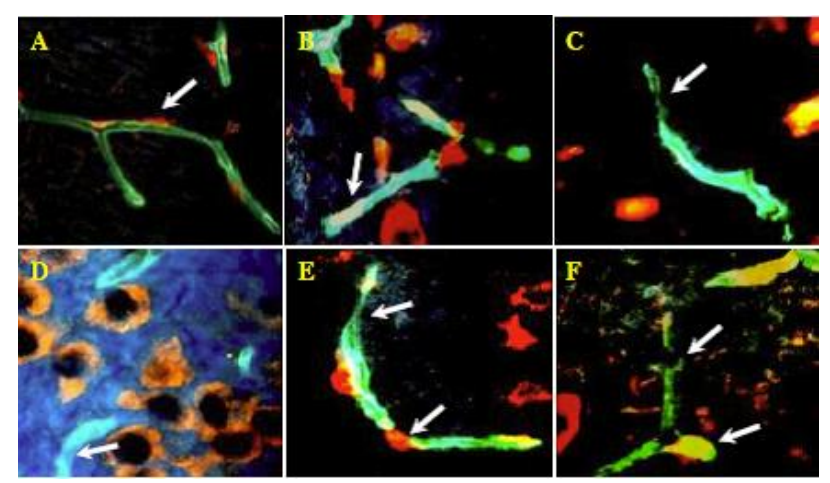

Figure 1. Effects of ozone treatment on Blood Brain Barrier (green) and Guanylyl cyclase (red) double immunofluorescence in the dentate gyrus of rats. Photomicrographs show not morphological alterations of endothelial cells and blood brain barrier (arrows) in control animals (A) and show s morphological alterations of endothelial cells and blood brain barrier (arrows) getting different times of ozone administration in the dentate gyrus of rats treated with air only (A), 7 days of ozone exposure (B), 15 days of ozone exposure (C), 30 days of ozone exposure (D), 60 days of ozone exposure (E), and 90 days of ozone exposure $(\mathrm{F})$. Magnification $100 \times, \mathrm{n}=6$ per group. 
astrocytes processes as well as an increase in the thickness and of the astrocytes foot length, which tend to wrap around the damaged vascular endothelium as time passes to ozone exposure. We can observe an increase in the $\mathrm{NF} \kappa$-B expression in the nucleus of endothelial cells from 15 days on of exposition of ozone until 90 days of exposure to this gas. The results show an increase in TNF- $\alpha$ expression of endothelial cells from 7 days on of exposure to ozone and also in capillary endothelium from 15 days on of treatment, which shows an increase at 15 and 30 days of exposure.

\section{DISCUSSION}

Previous studies conducted in our laboratory showed that chronic exposure to low doses of ozone in healthy animals without other factor added causes a chronic state of oxidative stress [6] which produces a progressive neurodegeneration process in the hippocampus of rats exposed to this gas. This process is characterized by a neuronal cell damage which provoke cellular death accompanied by lack of microglia, astrocytes and mitochondrial activation [6,7], loss of the regulation of inflammatory response and increased oxidative stress that causes to form a vicious cycle which maintains the neurodegenerative process.

We can establish that all the changes above mentioned are accompanied by an alteration of the BBB as shown in Figures 1(B)-(F) where we can observe that the small capillaries found in the hippocampal dentate gyrus exhibit continuity loss, edema of the endothelial cell and alterations in vascular endothelium itself, accompanied by glial and astrocytosis activation (Figures 2(B) and (C)).

We can also observe that exposure to low doses of ozone induces thickening of astrocytes foot and processes that targets to capillary endothelial cells (Figures 3(A.A)-(F.A)). Therefore we have a rupture of the BBB, endothelial changes, edema of the endothelial cell and an increase in nuclear volume (Figures 1(B)-(F) and
3(A.B)-(F.B), 3(A.C)-(E.C)) accompanied by thickening of endothelium wall, product of astrocytes stimulation. The results retrieved (Figures 3(A.B)-(F.B), and 3(A.C)(E.C)) also indicate that these changes are accompanied by alteration of the inflammatory response, in these results we can observe changes in the expression of factor $\mathrm{NF} \kappa$-B and also the expression of TNF- $\alpha$. All the above indicates that in the process of progressive neurodegeneration caused by exposure to low doses of ozone within neuronal death, glial activation presents a loss of selective permeability of the $\mathrm{BBB}$, this fact is of vital importance when we consider that $\mathrm{BBB}$ has the function of protecting the brain tissue and for this purpose its operation is very complex since it must maintain the normal homeostasis of the central nervous system through selective transport metabolism of substances present in blood and brain and moreover has numerous mitochondria and efficient enzymatic system [12]. Several pathologies are associated with an increase in the permeability of the BBB (ischemia and osmotic shock, infections and inflammatory processes that cause neurodegenerative diseases) $[11,14]$. Alterations in the permeability of the $\mathrm{BBB}$ is correlated with an increase of cytokines in blood and cerebrospinal fluid, with a raised TNF- $\alpha$ TNwhich also induces the expression of IL-Ib Et-L6 which likewise contribute to increase the permeability of the barrier. The experimental results of this work show that coupled with the rupture of BBB Figure 1 also increases the expression of NF $\kappa$-B and TNF- $\alpha$ in endothelial cells.

Further studies are needed to enhance and complement these finding, since it is very important to understand the involvement of oxidative stress in the blood-brain barrier disruption, and how these factors are participating in the development and progression of neurodegenerative diseases.

\section{CONCLUSION}

Exposure to low levels of ozone causes a chronic state of
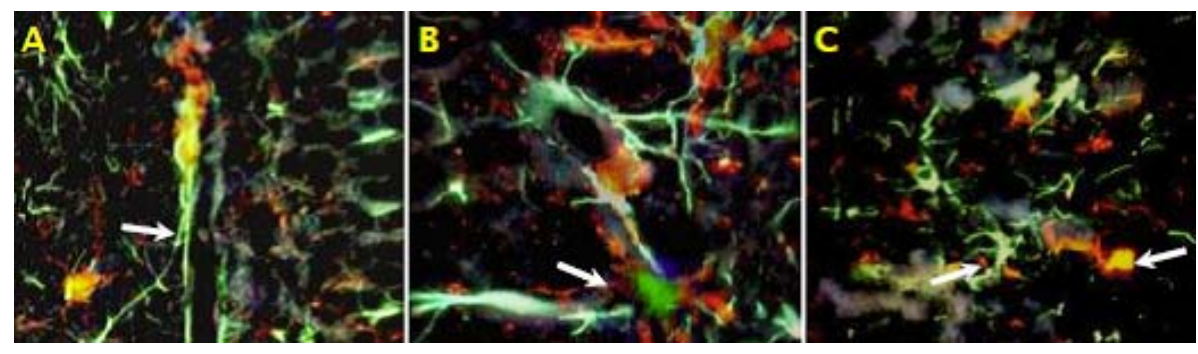

Figure 2. Effects of ozone treatment on GFAP (green) and Iba-1 (red) double immunofluorescence in the hippocampus. Photomicrographs show immunoreactivity in dentate gyrus of rats treated with air only, arrows indicates an astrocyte surrounding a normal blood vessel (A). 30 days of ozone exposure, morphological alterations of astrocytes surrounding an abnormal blood vessel (arrows) (B), 60 days of ozone exposure, observe GFAP (right arrow) and Iba-1 (left arrow) hyperactivity and in 60 days treatment in the dentate gyrus of this animals $(\mathrm{C})$. Magnification $100 \times, \mathrm{n}=6$ per group. 


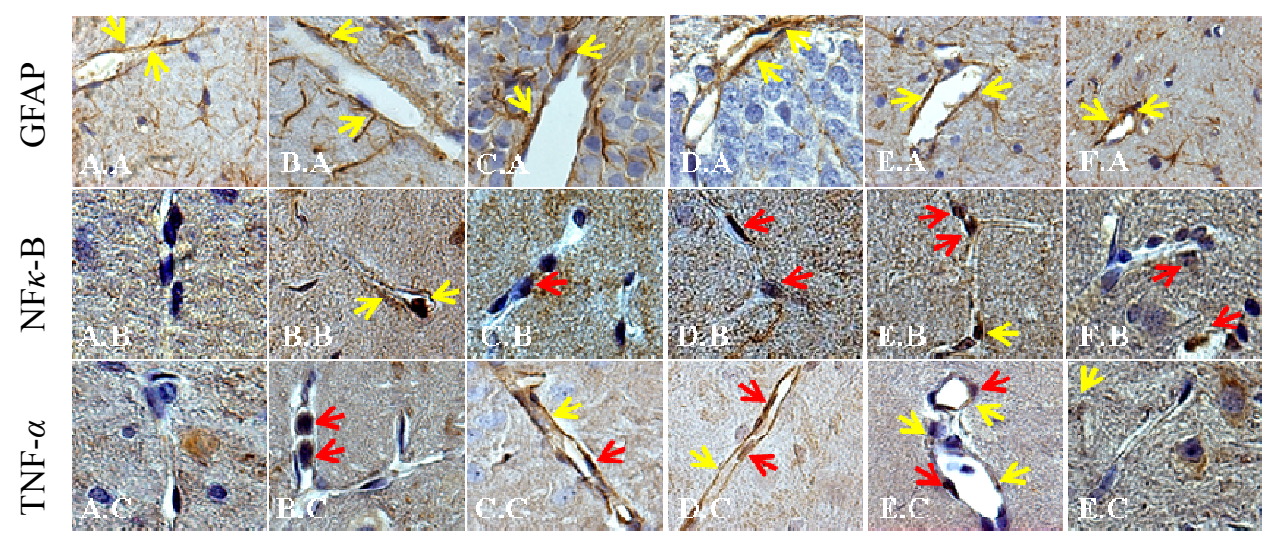

Figure 3. Effects of ozone treatment on GFAP, NF $\kappa-\mathrm{B}$ and TNF- $\alpha$ immunoreactivity in the rat hippocampus. Light photomicrographs show GFAP immunoreactivity in the dentate gyrus of rats treated with air only (A.A), 7 days of ozone exposure (B.A), 15 days of ozone exposure (C.A), 30 days of ozone exposure (D.A), 60 days of ozone exposure (E.A) and 90 days of exposure (F.A). Observe the increased cell immunoreactivity of GFAP in astrocytes surrounding blood vessels in dentate gyrus starting at 15 days of ozone treatments and ozone long exposures (arrows) (B.A-F.A). Observe NF $\kappa$-B normal expression in endothelial cells (red arrows) on tissue control (A.B.) and increased expression in endothelial cells of 7 days of ozone exposure (B.B,), 15 days of ozone exposure (C.B), 30 days of ozone exposure (D.B), 60 days of ozone exposure (E.B) and 90 days of exposure (F.B) (red arrows). In addition, observe some edematized endothelial cells (C.B, D.B, and F.B.). TNF- $\alpha$ expression in endothelial cells without ozone treatment (A.C) and increased expression in endothelial cells of 7 days of ozone exposure (B.C), 15 days of ozone exposure (C.C), 30 days of ozone exposure (D.C), 60 days of ozone exposure (E.C) and 90 days of exposure (F.C). Also observe some edematized endothelial cells (Arrows) in B.C, E.C. and E.C. Magnification $100 \times, \mathrm{n}=6$ per group.

oxidative stress which produces rupture of BBB with thickened astrocytes foot accompanied by edema of endothelial cells and an increase in the expression of inflammatory cytokines by the endothelium. All the above forms part of a progressive neurodegeneration process which forms a vicious circle that leads to a loss of control of cerebral repair processes and a slow increase in cell death.

\section{ACKNOWLEDGEMENTS}

This work was supported by the Dirección General de Apoyo al Personal Académico [Grant numbers: IN219511 to S. R-A].

\section{REFERENCES}

[1] Sierra-Vargas, M.P. and Teran, L.M. (2012) Air pollution: Impact and prevention. Respirology, 17, 1031-1038. http://dx.doi.org/10.1111/j.1440-1843.2012.02213.x

[2] Billionnet, C., Sherrill, D., Annesi-Maesano, I., GERIE, S. (2012) Estimating the health effects of exposure to multipollutant mixture. Annals of Epidemiology, 22, 126-141. http://dx.doi.org/10.1016/j.annepidem.2011.11.004

[3] Grella, E., Paciocco, G., Caterino, U. and Mazzarella, G. (2002) Respiratory function and atmospheric pollution. Monaldi Archives for Chest Disease, 57, 196-199.

[4] Delzell Jr., J.E. (2013) Common lung conditions: Environmental pollutants and lung disease. FP Essentials, 409,
$32-42$.

[5] Pope, C.A., Verrier, R.L., Lovett, E.G., Larson, A.C., Raizenne, M.E., Kanner, R.E., Schwartz, J., Villegas, G.M., Gold, D.R. and Dockery, D.W. (1999) Heart rate variability associated with particulate air pollution. American Heart Journal, 138, 890-899. http://dx.doi.org/10.1016/S0002-8703(99)70014-1

[6] Rivas-Arancibia, S., Guevara-Guzmán, R., López-Vidal, Y., Rodríguez-Martínez, E., Zanardo-Gomes, M., AngoaPérez, M. and Raisman-Vozari, R. (2010) Oxidative stress caused by ozone exposure induces loss of brain repair in the hippocampus of adult rats. Toxicological Science, 113,187-197.

[7] Rodríguez-Martínez, E., Martínez, F., Espinosa-García, M.T., Maldonado, P. and Rivas-Arancibia, S. (2013) Mitochondrial dysfunction in the hippocampus of rats caused by chronic oxidative stress. Neuroscience, 252, 384-395. http://dx.doi.org/10.1016/i.neuroscience.2013.08.018

[8] Iles, K.E. and Jay, F. (2002) Macrophage signaling and respiratory burst. Immunological Research, 26, 95-105. http://dx.doi.org/10.1385/IR:26:1-3:095

[9] Valko, M., Leibfritz, D., Moncol, J., Cronin, M.T., Mazur, M. and Telser, J. (2007) Free radicals and antioxidants in normal physiological functions and human disease. International Journal of Biochemistry and Cell Biology, 39, 44-84.

[10] Banks, W.A. and Erickson, M.A. (2010) The blood-brain barrier and immune function and dysfunction. Neurobiology of Disease, 37, 26-32. http://dx.doi.org/10.1016/j.nbd.2009.07.031 
[11] Stolp, H.B., Liddelow, S.A., Sá-Pereira, I., Dziegielewska, K.M. and Saunders, N.R. (2013) Immune responses at brain barriers and implications for brain development and neurological function in later life. Frontiers in Integrative Neuroscience, 7, 61 . http://dx.doi.org/10.3389/fnint.2013.00061

[12] Enciu, A.M., Gherghiceanu, M. and Popescu, B.O. (2013) Triggers and effectors of oxidative stress at blood-brain barrier level: Relevance for brain ageing and neurodegeneration. Oxidative Medicine and Cellular Longevity, 2013, 297512. http://dx.doi.org/10.1155/2013/297512
[13] Dorheim, M.A., Tracey. W.R., Pollock, J.S. and Grammas, P. (1994) Nitric oxide synthase activity is elevated in brain microvessels in Alzheimer's disease. Biochemical and Biophysical Research Communications, 30, 659665. http://dx.doi.org/10.1006/bbrc.1994.2716

[14] Erickson, M.A. and Banks, W.A. (2013) Blood-brain barrier dysfunction as a cause and consequence of Alzheimer's disease. Journal of Cerebral Blood Flow \& Metabolism, 33, 1500-1513.

http://dx.doi.org/10.1038/jcbfm.2013.135 